\title{
Intake of wholegrain products is associated with dietary, lifestyle, anthropometric and socio-economic factors in Denmark
}

\author{
Rikke Egeberg ${ }^{1, *}$, Kirsten Frederiksen ${ }^{1}$, Anja Olsen ${ }^{1}$, Nina F Johnsen ${ }^{1}$, Steffen Loft ${ }^{2}$, \\ Kim Overvad ${ }^{3}$ and Anne Tjønneland ${ }^{1}$ \\ 'Institute of Cancer Epidemiology, The Danish Cancer Society, Strandboulevarden 49, DK-2100 Copenhagen, \\ Denmark: ${ }^{2}$ Institute of Public Health, University of Copenhagen, Copenhagen, Denmark: ${ }^{3}$ Department of Clinical \\ Epidemiology, Aarhus University Hospital, Aalborg, Denmark
}

Submitted 28 January 2008: Accepted 18 November 2008: First published online 6 February 2009

\begin{abstract}
Objective: To evaluate the association between wholegrain products intake and other dietary, lifestyle, anthropometric and socio-economic factors.

Design: Cross-sectional study, with data on diet, lifestyle and socio-economic factors obtained from questionnaires. Anthropometric measurements were collected by trained professionals. Multiple linear and principal components regression analyses were used in statistical analyses.

Setting: Part of the Diet, Cancer and Health study, a prospective cohort study to evaluate the aetiological role of diet on cancer risk, conducted in the greater Copenhagen and Aarhus area, Denmark.

Subjects: Men and women ( $n$ 54720) aged 50-64 years.

Results: In multiple linear regression analyses focusing on individual dietary factors, intake of wholegrain products was associated with intake of all dietary factors studied (fish, red meat, poultry, processed meat, dairy products, fruits, vegetables, cakes and refined-grain products). The strongest positive associations were seen for intake of vegetables and processed meat, whereas the strongest negative associations were seen for intake of red meat and refined-grain products. Regression analyses on dietary patterns identified by principal components analysis yielded similar results. Also, wholegrain products intake was positively associated with cycling, taking dietary supplements and high school education, and negatively associated with intake of alcohol, BMI and smoking.

Conclusions: Intake of wholegrain products is associated with other dietary factors, healthier lifestyle habits and higher socio-economic status. Therefore future studies need to account for the possible confounding by other dietary and lifestyle-related parameters when investigating relationships between wholegrain products intake and disease risk.
\end{abstract}

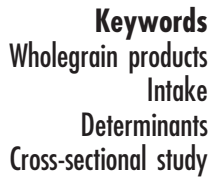

In recent years, there has been considerable international interest in the effect of whole grains in relation to mortality and a number of chronic diseases. Prospective studies have shown an inverse relationship between the consumption of wholegrain products or specific foods with a high content of whole grains and risk of total mortality ${ }^{(1-4)}, \mathrm{CVD}^{(2,5-9)}$, type 2 diabetes ${ }^{(10-14)}$ and certain cancers ${ }^{(15-17)}$. A high intake of wholegrain products has also been linked to lower $\mathrm{BMI}^{(18-21)}$, lower gain in weight or $\mathrm{BMI}^{(22-24)}$ and lower risk of presence of the metabolic syndrome ${ }^{(25-27)}$, which is described as a cluster of risk factors that is related to the onset of CVD, type 2 diabetes and perhaps also specific cancers. Although the evidence is not entirely consistent, there seems to exist a protective role of wholegrain products and it has been highly indicated that intake of wholegrain products is of considerable importance for public health.

Whole grains contribute a range of beneficial nutrients including dietary fibres, vitamins, minerals and phytochemicals, and the possibly protective effect of whole grains may be mediated via the effect of these compounds ${ }^{(28)}$. However, increased knowledge of the biological aspects of whole grains is needed to gain full insights into the health potential of wholegrain products. Whether the beneficial effects of wholegrain products indicated by existing research are due to a direct effect of the wholegrain products and/or simply to the effect of confounding is another important issue. Other factors 
might confound an association between consumption of wholegrain products and disease, thus the associations observed in studies supporting a protective effect of wholegrain products may simply reflect the effect of other dietary or non-dietary factors and not the causal association between wholegrain products and disease. Earlier studies have indicated that high consumption of whole grains is associated with healthier dietary and lifestyle habits $^{(3,29-31)}$. It is possible that these associations may vary between populations because of differences in food and lifestyle habits.

To further clarify the effect of wholegrain products in relation to risk of diseases, it is important to understand the association between intake of wholegrain products and other risk factors of diseases for accurate interpretation of the increasing number of studies relating high intake of wholegrain products to lower risk of chronic diseases. The aim of the present study was to evaluate the association between intake of wholegrain products and other dietary, lifestyle, anthropometric and socioeconomic factors.

\section{Methods}

\section{Study population and design}

The Diet, Cancer and Health study is a prospective cohort study established with the primary purpose to evaluate the aetiological role of diet on cancer risk. A more detailed description of the cohort has been published elsewhere ${ }^{(32)}$. In brief, from December 1993 to May 1997, all men and women living in the greater Copenhagen and Aarhus area and fulfilling the following inclusion criteria: age between 50 and 64 years, born in Denmark and not registered with a previous cancer diagnosis in the Danish Cancer Registry, were invited to participate in the study (80996 men and 79729 women). Subjects were identified by the unique 10-digit identification number, which is allocated to every Danish citizen by the Central Population Registry. Of the invited persons, 27178 men (33.6\% of those invited) and 29875 women $(37 \cdot 5 \%$ of those invited) participated in the study. All participants attended one of two established study centres in Copenhagen or Aarhus. The Diet, Cancer and Health study and the present study were approved by the Regional Ethical Committees on human studies in Copenhagen and Aarhus, and by the Danish Data Protection Agency.

\section{Dietary information}

A validated 192-item semi-quantitative FFQ completed at baseline was used for estimating the participants' habitual diet during the preceding 12 months ${ }^{(33-36)}$. Consumption was assessed in twelve categories of predefined responses, ranging from 'never' to ' 8 times or more per day'. Daily intakes of foods and nutrients for each participant were calculated by the FOODCalc program (www.foodcalc.dk) using population-specific standardized recipes and portion sizes.

'Wholegrain products' comprised intake of rye bread, wholegrain bread, crisp bread, oatmeal and corn, and was measured in $\mathrm{g} / \mathrm{d}$. Other dietary factors of interest included 'red meat', 'poultry', 'processed meat', 'fish', 'lean dairy products', 'fatty dairy products', 'fruits', 'vegetables', 'cakes' and 'refined-grain products'. Intake of all dietary factors was measured in $\mathrm{g} / \mathrm{d}$. Intake of 'alcohol' was measured in $\mathrm{g} / \mathrm{d}$ and was assessed by summing the products of frequency of use of specific beverages (beer, wine, fortified wine and spirits) by their ethanol content. In the questionnaire, the participants indicated which type of fat they preferred for cooking, denoted as 'use of fatty substance when cooking'. Participants were grouped into three categories as vegetable oil users, margarine/ butter users or non-users of a fatty substance when cooking. A section of the FFQ asked for information on the use of dietary supplements. Participants were asked about the exact brand, type, frequency and doses of the dietary supplements they used. Three categories of 'use of dietary supplements' were created. Participants were categorized as a supplement non-user, a multivitamin/ mineral supplement user (i.e. a person taking a multivitamin/mineral supplement only) or a user of any supplement (i.e. taking one or more supplements alone or in combination with a multivitamin/mineral supplement).

\section{Lifestyle and socio-economic factors}

A lifestyle questionnaire was used for collecting information on lifestyle habits, social factors, reproductive factors and health status. Information included in the present study pertained to smoking status, alcohol habits, physical activity during leisure time, use of hormone replacement therapy (HRT; women only) and school education. Alcohol habits were defined in two ways: (i) 'alcohol status', with the four categories of never drinkers, ex-drinkers, occasional drinkers and current drinkers; and (ii) 'alcohol preference', with the four categories of wine drinkers, beer drinkers, spirit drinkers and mixed drinkers. A participant's preference for a specific beverage type was based on whether the person's intake of that beverage constituted $\geq 50 \%$ of their total alcohol intake. If intake of none of the specific beverage types exceeded $50 \%$, the person was categorized as a mixed drinker. Participants were divided into three categories of 'smoking status': never smokers, past smokers and current smokers. Physical activity during leisure time was assessed by twelve questions covering the average number of hours per week spent in the past year on six types of activities: 'walking', 'cycling', 'sports', 'gardening', 'housework' and 'hobby work', during summer and winter, respectively. The number of hours spent on physical activity during summer and winter were averaged. Women were divided into three categories of 'HRT use': never users, past users and current 
users. Socio-economic status was defined based on the participant's school education. Participants were divided into three categories of 'school education': low school education ( $\leq 7$ years), medium school education (8-10 years) and high school education ( $\geq 11$ years).

\section{Anthropometric measurements}

All participants were measured in light underwear. At baseline, weight, height and waist circumference were measured for every participant at the study centres by trained professionals. BMI was calculated as [weight $(\mathrm{kg})] /[\text { height }(\mathrm{m})]^{2}$. Waist circumference was measured at the smallest horizontal circumference between the ribs and iliac crest (the natural waist) or, in case of an indeterminable waist narrowing, halfway between the lower rib and the iliac crest. Waist circumference was measured to the nearest half centimetre.

\section{Definition of the study population for analysis}

Of the 57053 participants, 569 were excluded because of a cancer diagnosis reported to the Danish Cancer Registry before entry into the study. Additionally, thirty-seven were excluded because they failed to fill in the lifestyle questionnaire and 1382 were excluded because of unrealistic or missing values in the dietary, lifestyle, anthropometric or socio-economic variables. Finally, fiftyfive and 290 were excluded because of missing values or a null intake of wholegrain products, respectively. Thus 54720 participants (26 175 men and 28545 women) were included in the analyses.

\section{Statistics}

The descriptive characteristics are presented as median values with 5th and 95th percentiles for continuous variables and categorical variables are presented as percentages. The associations between intake of wholegrain products and dietary, lifestyle, anthropometric and socioeconomic variables were investigated in multiple linear regression analyses. Intake of wholegrain products measured in $\mathrm{g} / \mathrm{d}$ was the dependent variable. Associations were investigated in models including age, centre (Copenhagen and Aarhus), dietary variables (fish, red meat, poultry, processed meat, lean dairy products, fatty dairy products, fruits, vegetables, refined-grain products and cakes), lifestyle variables (alcohol intake, alcohol status, alcohol preference, smoking status, physical activity, use of fatty substance when cooking, use of dietary supplements and use of HRT), anthropometric variables (BMI and waist circumference) and socio-economic variables (school education). Age, all dietary variables, BMI, waist circumference and all physical activity variables were entered as continuous variables. Smoking status, alcohol status, alcohol preference, use of fatty substance when cooking, use of dietary supplements and use of HRT were entered as categorical variables with the categories previously described.
For all continuous variables the assumption of linearity was evaluated by linear splines with three or nine boundaries placed at the quartiles (25th, 50th and 75th) or centiles (10th to 90th) of the distribution ${ }^{(37)}$. The six physical activity variables (walking, cycling, sports, housework, gardening and hobby work) were log-transformed and showed no signs of deviation from linearity when evaluated by linear splines with three boundaries. For the dietary variables (fish, red meat, poultry, processed meat, lean dairy products, fatty dairy products, fruits, vegetables, refined-grain products and cakes), BMI, waist circumference and alcohol intake, the assumption of linearity was not appropriate and in these cases the estimates and tests should be interpreted with caution. To take into account that the assumption of linearity was not met for specific variables a separate model for each of the variables entering the model as a linear variable was made, with all other linear variables entering the model as spline parameters. All linear variables were scaled to reflect realistic and practical increments in the daily intake of the food items (based on the interquartile range).

Principal components analysis was applied to extract dietary patterns from ten food groups (fish, red meat, poultry, processed meat, lean dairy products, fatty dairy products, fruits, vegetables, refined-grain products and cakes) using the correlation matrix. The main constituents of the ten dietary patterns (components) extracted are shown in Appendix 1 (women) and Appendix 2 (men). Scoring coefficients indicate the degree of correlation between the original variables (residuals of food group intakes) and the components extracted. A positive scoring coefficient indicates that the original dietary variable is positively associated with the particular component, while a negative scoring coefficient implies a negative association. Multiple linear regression analysis was applied to model the associations between intake of wholegrain products and dietary patterns calculated from the principal components analysis. The ten components were included in the model simultaneously, with additional adjustments for age, centre and all lifestyle, anthropometric and socio-economic variables studied.

The GLM and PRINCOMP procedures in the SAS statistical software package release 9.0 (SAS Institute Inc., Cary, NC, USA) on a TextPad platform were used for the statistical analyses.

\section{Results}

The baseline characteristics of the participants are shown in Table 1 . The median age at entry into the study for the 54720 participants included was 56 years (range 50-64 years). The median intake of wholegrain products was $140 \mathrm{~g} / \mathrm{d}$ among men and $119 \mathrm{~g} / \mathrm{d}$ among women (Table 2). Rye bread contributed to $63 \%$ and $55 \%$ of the total wholegrain products intake among men and women, respectively (Table 2 ). 
Table 1 Baseline characteristics of participants included in the Diet, Cancer and Health cohort study (1993-7)

\begin{tabular}{|c|c|c|c|c|c|c|}
\hline & \multicolumn{3}{|c|}{ Men ( $n 26$ 175) } & \multicolumn{3}{|c|}{ Women (n 28545) } \\
\hline & Median & P5 & P95 & Median & P5 & P95 \\
\hline \multicolumn{7}{|l|}{ Demographic variables } \\
\hline Age (years) & 56 & 50 & 64 & 56 & 50 & 64 \\
\hline \multicolumn{7}{|l|}{ Centre } \\
\hline Aarhus (\%) & 32 & & & 30 & & \\
\hline Copenhagen (\%) & 68 & & & 70 & & \\
\hline \multicolumn{7}{|l|}{ Dietary variables } \\
\hline Fish $(g / d)$ & 42 & 13 & 99 & 35 & 11 & 85 \\
\hline Red meat $(\mathrm{g} / \mathrm{d})$ & 100 & 46 & 190 & 63 & 27 & 121 \\
\hline Poultry (g/d) & 20 & 5 & 62 & 16 & 4 & 56 \\
\hline Processed meat $(\mathrm{g} / \mathrm{d})$ & 35 & 9 & 89 & 18 & 4 & 50 \\
\hline Lean dairy products (g/d) & 163 & 19 & 922 & 206 & 20 & 764 \\
\hline Fatty dairy products $(\mathrm{g} / \mathrm{d})$ & 63 & 18 & 515 & 65 & 20 & 316 \\
\hline Fruits $(\mathrm{g} / \mathrm{d})$ & 143 & 23 & 468 & 202 & 38 & 567 \\
\hline Vegetables $(\mathrm{g} / \mathrm{d})$ & 151 & 47 & 341 & 172 & 51 & 389 \\
\hline Cakes $(\mathrm{g} / \mathrm{d})$ & 14 & 2 & 67 & 13 & 2 & 57 \\
\hline Refined grain products $(\mathrm{g} / \mathrm{d})$ & 55 & 18 & 140 & 38 & 13 & 103 \\
\hline \multicolumn{7}{|l|}{ Lifestyle variables } \\
\hline \multicolumn{7}{|c|}{ Use of fatty substance when cooking } \\
\hline None (\%) & $0 \cdot 3$ & - & - & $0 \cdot 3$ & - & - \\
\hline Vegetables oils (\%) & 28 & - & - & 30 & - & - \\
\hline Margarine/butter (\%) & 72 & - & - & 70 & - & - \\
\hline \multicolumn{7}{|l|}{ Use of dietary supplements } \\
\hline Non-user (\%) & 37 & - & - & 24 & - & - \\
\hline Multivitamin/mineral user (\%) & 18 & - & _- & 15 & - & _- \\
\hline Any user (\%) & 45 & - & - & 61 & - & - \\
\hline Alcohol intake* $(\mathrm{g} / \mathrm{d})$ & 20 & 2 & 80 & 10 & 1 & 42 \\
\hline \multicolumn{7}{|l|}{ Alcohol status } \\
\hline Never drinker (\%) & $0 \cdot 2$ & - & - & 1 & - & - \\
\hline Ex-drinker (\%) & $1 \cdot \overline{3}$ & - & _- & 1 & - & _- \\
\hline Occasional drinker (\%) & 1 & - & - & 2 & - & - \\
\hline Current drinker (\%) & 97 & - & - & 96 & _ & _ \\
\hline \multicolumn{7}{|l|}{ Alcohol preference ${ }^{*}$} \\
\hline Wine drinker (\%) & 34 & - & - & 66 & - & - \\
\hline Beer drinker (\%) & 42 & - & _- & 14 & - & _- \\
\hline Spirits drinker (\%) & 3 & - & - & 3 & - & - \\
\hline Mixed drinker (\%) & 19 & - & - & 14 & - & - \\
\hline \multicolumn{7}{|l|}{ Smoking status } \\
\hline Never smoker (\%) & 26 & - & - & 44 & - & - \\
\hline Past smoker (\%) & 35 & - & - & 24 & - & - \\
\hline Current smoker (\%) & 39 & - & - & 33 & - & - \\
\hline \multicolumn{7}{|l|}{ Hormone replacement therapy use } \\
\hline Never user (\%) & - & - & - & 55 & - & - \\
\hline Past user (\%) & - & - & - & 16 & - & - \\
\hline Current user (\%) & - & - & - & 30 & - & - \\
\hline BMI $\left(\mathrm{kg} / \mathrm{m}^{2}\right)$ & 26 & 21 & 33 & 25 & 20 & 34 \\
\hline Waist circumference $(\mathrm{cm})$ & 95 & 81 & 114 & 80 & 67 & 103 \\
\hline \multicolumn{7}{|l|}{ Recreational activity } \\
\hline Walking (h/week) & 3 & 1 & 12 & 3 & 1 & 12 \\
\hline Cycling (h/week) & 2 & 0.5 & 10 & 2 & 0.5 & 10 \\
\hline Sports (h/week) & 2 & 0.5 & $7 \cdot 5$ & $1 \cdot 5$ & 0.5 & 6 \\
\hline \multicolumn{7}{|l|}{ Household activity } \\
\hline Housework (h/week) & 2 & 1 & 10 & 5 & 2 & 20 \\
\hline Gardening (h/week) & 2 & 0.5 & 10 & 2 & 0.5 & 8 \\
\hline Hobby work (h/week) & 2 & 1 & 11 & 1 & 0.5 & 7 \\
\hline \multicolumn{7}{|l|}{ Socio-economic variables } \\
\hline Education & & & & & & \\
\hline Low (\%) & 34 & - & - & 31 & - & - \\
\hline Medium (\%) & 42 & - & - & 50 & - & - \\
\hline High (\%) & 24 & - & - & 19 & - & - \\
\hline
\end{tabular}

P5, 5th percentile; P95, 95th percentile.

*Among current drinkers.

Table 3 (women) and Table 4 (men) show the univariate and mutual adjusted regression coefficients and corresponding 95\% confidence intervals for the regression of wholegrain products $v$. dietary, physical activity and other lifestyle, anthropometric and socio-economic factors. In the univariate analyses with adjustment for age and centre, intake of wholegrain products was significantly associated with all dietary variables studied 
Table 2 Baseline intakes of total grain products, wholegrain products and rye bread of participants included in the Diet, Cancer and Health cohort study (1993-7)

\begin{tabular}{|c|c|c|c|c|c|c|}
\hline & \multicolumn{3}{|c|}{ Men (n 26175$)$} & \multicolumn{3}{|c|}{ Women (n 28545) } \\
\hline & Median & P5 & P95 & Median & P5 & P95 \\
\hline Total grain products $(\mathrm{g} / \mathrm{d})$ & 210 & 100 & 356 & 166 & 80 & 290 \\
\hline Wholegrain products ( $\mathrm{g} / \mathrm{d}$ ) & 140 & 48 & 279 & 119 & 40 & 237 \\
\hline$\%$ of the total grain intake & 71 & - & - & 76 & - & - \\
\hline Rye bread $(\mathrm{g} / \mathrm{d})$ & 63 & 20 & 163 & 63 & 11 & 113 \\
\hline$\%$ of the whole grain intake & 63 & - & - & 55 & - & - \\
\hline
\end{tabular}

P5, 5th percentile; P95, 95th percentile.

among both men and women. All physical activity and other lifestyle, anthropometric and socio-economic variables studied, except hours per week spent walking or gardening among men and hours per week spent doing housework or hobby work and HRT use among women, were in addition significantly associated with intake of wholegrain products in univariate analyses. Among both men and women, mutual adjustments weakened the associations for the most of the dietary variables, except for intake of red meat, processed meat and refined-grain products, where the estimated associations were strengthened. Among the dietary variables, intake of wholegrain products was strongest positively associated with intake of processed meat and vegetables and strongest negatively associated with intake of red meat and refined-grain products after mutual adjustments. Regarding the physical activity variables mutual adjustments weakened all the estimated associations, resulting in only hours per week spent cycling being significantly positively associated with intake of wholegrain products among both men and women. For the remaining lifestyle, anthropometric and socio-economic variables, mutual adjustments resulted in that intake of wholegrain products among women was positively associated with being a multivitamin/mineral user, being an ex-drinker and medium or high school education, but negatively associated with intake of alcohol, BMI and current smoking. Among men, intake of wholegrain products was also positively associated with being a multivitamin/ mineral user or any user of dietary supplements and high school education, but negatively associated with intake of alcohol, BMI and waist circumference, use of margarine/ butter when cooking, a preference for drinking beer and current smoking after mutual adjustments.

Twelve per cent (women) and 15\% (men) of the variation in the wholegrain products intake was explained by dietary variables. Taking the physical activity variables into consideration, 13\% (women) and 17\% (men) of the variation was explained by dietary and physical activity variables combined. Overall, the multiple regression model explained $16 \%$ and $21 \%$ of the variance in the wholegrain products intake among women and men, respectively (Tables 3 and 4).

Table 5 shows the mutually adjusted regression coefficients and their corresponding standard errors and
$P$ values of the ten principal components extracted from the principal components analysis. Among men, the strongest positive association with intake of wholegrain products was seen for component $10(\beta=21 \cdot 6$, SE $0 \cdot 6)$, which loaded positively on processed meat and vegetables and negatively on red meat and refined-grain products. This component accounted for $6 \%$ of the variation in the total intake of wholegrain products. Intake of wholegrain products was also highly positively associated with component $1(\beta=8 \cdot 4$, se $0 \cdot 3)$, which loaded positively on all dietary factors studied, and component 5 $(\beta=10 \cdot 8$, se $0 \cdot 4)$, which was characterized by intake of dairy products and fruits. The strongest negative association was seen for component $2(\beta=-4 \cdot 9$, se $0 \cdot 4)$. This component loaded positively on red meat, processed meat, cakes and refined-grain products and negatively on fish, poultry, fruits and vegetables. Among women, the strongest positive association with intake of wholegrain products was seen for component $1(\beta=7 \cdot 1$, SE $0 \cdot 2)$, which loaded positively on all dietary factors and accounted for $3 \%$ of the variation in the total intake of wholegrain products. Component 10 was also highly positively associated with wholegrain products intake $(\beta=13 \cdot 6$, SE 0 .5). This component loaded positively on processed meat and vegetables and negatively on red meat. The strongest negative association was seen for component 2 $(\beta=-5 \cdot 1$, SE $0 \cdot 30)$, which loaded positively on red meat, processed meat, cakes and refined-grain products and negatively on fish, poultry, fruits and vegetables.

\section{Discussion}

In the present study we found that intake of wholegrain products is associated with several dietary, lifestyle, anthropometric and socio-economic factors in a cohort of Danish middle-aged men and women with a high consumption of this food group. Among both men and women the intake of wholegrain products was positively associated with other dietary factors, especially intake of processed meat and vegetables, and negatively associated with intake of red meat and refined-grain products. In addition, positive associations with more hours of cycling per week, taking dietary supplements and high 
Table 3 Regression derived coefficients $(\beta)$ and $95 \%$ confidence intervals of dietary, lifestyle, anthropometric and socio-economic variables among 28545 women included in the Diet, Cancer and Health cohort study (1993-7)

\begin{tabular}{|c|c|c|c|c|c|}
\hline & \multicolumn{2}{|c|}{ Analysis adjusted for age and centre } & \multicolumn{2}{|c|}{ Mutual adjusted analysis } & \multirow[b]{2}{*}{$R^{2}$} \\
\hline & $\beta^{*}$ & $95 \% \mathrm{Cl}$ & $\beta^{*}$ & $95 \% \mathrm{Cl}$ & \\
\hline \multicolumn{5}{|l|}{ Dietary variables } & $0 \cdot 12 \S$ \\
\hline Fish (per $25 \mathrm{~g}$ ) & $8 \cdot 9$ & $8 \cdot 2,9 \cdot 6$ & $3 \cdot 0$ & $2 \cdot 3,3 \cdot 8$ & \\
\hline Red meat (per $50 \mathrm{~g}$ ) & $-2 \cdot 6$ & $-3 \cdot 8,-1 \cdot 4$ & $-10 \cdot 6$ & $-11 \cdot 8,-9 \cdot 3$ & \\
\hline Poultry (per $10 \mathrm{~g}$ ) & $3 \cdot 7$ & $3 \cdot 3,4 \cdot 1$ & $1 \cdot 8$ & $1 \cdot 4,2 \cdot 2$ & \\
\hline Processed meat (per $10 \mathrm{~g}$ ) & $2 \cdot 6$ & $2 \cdot 1,3 \cdot 1$ & $5 \cdot 8$ & $5 \cdot 4,6 \cdot 3$ & \\
\hline Lean dairy products (per $100 \mathrm{~g}$ ) & $2 \cdot 4$ & $2 \cdot 1,2 \cdot 6$ & $1 \cdot 3$ & $1 \cdot 1,1 \cdot 6$ & \\
\hline Fatty dairy products (per $50 \mathrm{~g}$ ) & $2 \cdot 1$ & $1 \cdot 8,2 \cdot 4$ & $1 \cdot 4$ & $1 \cdot 2,1 \cdot 7$ & \\
\hline Fruits (per $100 \mathrm{~g}$ ) & $4 \cdot 6$ & $4 \cdot 2,5 \cdot 1$ & $0 \cdot 3$ & $-0 \cdot 1,0 \cdot 8$ & \\
\hline Vegetables (per $100 \mathrm{~g}$ ) & $13 \cdot 6$ & $12 \cdot 9,14 \cdot 2$ & $8 \cdot 8$ & $8 \cdot 1,9 \cdot 5$ & \\
\hline Cakes (per $10 \mathrm{~g}$ ) & $2 \cdot 5$ & $2 \cdot 1,2 \cdot 8$ & $3 \cdot 0$ & $2 \cdot 7,3 \cdot 4$ & \\
\hline Refined grain products (per $30 \mathrm{~g}$ ) & $-6 \cdot 2$ & $-7 \cdot 0,-5 \cdot 5$ & $-10 \cdot 7$ & $-11 \cdot 5,-10 \cdot 0$ & \\
\hline \multicolumn{5}{|l|}{ Physical activity variables } & $0 \cdot 13 \|$ \\
\hline Walking (per $1 \mathrm{~h}$ ) & $2 \cdot 3$ & $0 \cdot 2,4 \cdot 4$ & $-1 \cdot 3$ & $-3 \cdot 4,0 \cdot 7$ & \\
\hline Cycling (per $1 \mathrm{~h})$ & $11 \cdot 6$ & $9 \cdot 4,13 \cdot 8$ & $7 \cdot 8$ & $5 \cdot 7,9 \cdot 8$ & \\
\hline Sports (per $1 \mathrm{~h})$ & $5 \cdot 2$ & $2 \cdot 3,8 \cdot 1$ & $-2 \cdot 2$ & $-4 \cdot 9,0 \cdot 5$ & \\
\hline Housework (per $1 \mathrm{~h}$ ) & 0.3 & $-1 \cdot 8,2 \cdot 5$ & $-0 \cdot 1$ & $-2 \cdot 1,2 \cdot 2$ & \\
\hline Gardening (per $1 \mathrm{~h}$ ) & $3 \cdot 6$ & $1 \cdot 3,5 \cdot 9$ & $2 \cdot 1$ & $-0 \cdot 1,4 \cdot 3$ & \\
\hline Hobby work (per $1 \mathrm{~h})$ & 0.5 & $-2 \cdot 9,3 \cdot 8$ & $-0 \cdot 4$ & $-3 \cdot 6,2 \cdot 7$ & \\
\hline \multicolumn{5}{|l|}{ Lifestyle and socio-economic variables } & $0 \cdot 16$ \\
\hline \multicolumn{6}{|l|}{ Use of fatty substance when cooking } \\
\hline None & Ref. & & Ref. & & \\
\hline Vegetable oil & $14 \cdot 9$ & $1 \cdot 0,28 \cdot 8$ & $9 \cdot 2$ & $-3 \cdot 6,21 \cdot 9$ & \\
\hline Margarine/butter & 0.2 & $-13 \cdot 7,14 \cdot 1$ & $3 \cdot 2$ & $-9 \cdot 6,15 \cdot 9$ & \\
\hline \multicolumn{6}{|l|}{ Use of dietary supplement } \\
\hline Non-user & Ref. & & Ref. & & \\
\hline Multivitamin/mineral user & $12 \cdot 3$ & $9 \cdot 9,14 \cdot 6$ & $6 \cdot 2$ & $4 \cdot 0,8 \cdot 3$ & \\
\hline Any user & $14 \cdot 8$ & $13 \cdot 1,16 \cdot 5$ & $6 \cdot 4$ & $4 \cdot 8,8 \cdot 0$ & \\
\hline Alcohol intaket (per $10 \mathrm{~g}$ ) & $-2 \cdot 7$ & $-3 \cdot 2,-2 \cdot 3$ & -1.9 & $-2 \cdot 4,-1 \cdot 5$ & \\
\hline \multicolumn{6}{|l|}{ Alcohol status } \\
\hline Never drinker & Ref. & & Ref. & & \\
\hline Ex-drinker & $20 \cdot 0$ & $9 \cdot 7,30 \cdot 3$ & $11 \cdot 6$ & $2 \cdot 3,20 \cdot 9$ & \\
\hline Occasional drinker & $4 \cdot 3$ & $-5 \cdot 0,13 \cdot 6$ & $6 \cdot 2$ & $-4 \cdot 8,17 \cdot 2$ & \\
\hline Current drinkerł & $14 \cdot 0$ & $6 \cdot 4,21 \cdot 5$ & $11 \cdot 3$ & $-1 \cdot 3,23 \cdot 9$ & \\
\hline \multicolumn{6}{|l|}{ Alcohol preferencet } \\
\hline Wine drinker & Ref. & & Ref. & & \\
\hline Beer drinker & $-7 \cdot 7$ & $-9 \cdot 8,-5 \cdot 6$ & $-0 \cdot 6$ & $-2 \cdot 5,1 \cdot 4$ & \\
\hline Spirits drinker & $-6 \cdot 9$ & $-10.9,-3.0$ & $-1 \cdot 1$ & $-4 \cdot 7,2 \cdot 5$ & \\
\hline Mixed drinker & $-1 \cdot 1$ & $-3 \cdot 2,1 \cdot 0$ & $-0 \cdot 2$ & $-2 \cdot 2,1 \cdot 7$ & \\
\hline \multicolumn{6}{|l|}{ Smoking status } \\
\hline Never smoker & Ref. & & Ref. & & \\
\hline Past smoker & $-1 \cdot 3$ & $-3 \cdot 1,0 \cdot 5$ & $-0 \cdot 8$ & $-2 \cdot 5,0.9$ & \\
\hline Current smoker & $-18 \cdot 4$ & $-20 \cdot 0,-16 \cdot 7$ & $-10 \cdot 5$ & $-12 \cdot 1,-8 \cdot 9$ & \\
\hline \multicolumn{6}{|l|}{ Hormone replacement therapy use } \\
\hline Never user & Ref. & & Ref. & & \\
\hline Past user & $-2 \cdot 0$ & $-4 \cdot 1,0 \cdot 1$ & $-0 \cdot 4$ & $-2 \cdot 3,1 \cdot 4$ & \\
\hline Current user & $-0 \cdot 1$ & $-1 \cdot 7,1 \cdot 6$ & $-0 \cdot 8$ & $-2 \cdot 3,0 \cdot 7$ & \\
\hline BMI (per $1 \mathrm{~kg} / \mathrm{m}^{2}$ increment) & $-1 \cdot 1$ & $-1 \cdot 2,-0 \cdot 9$ & $-1 \cdot 3$ & $-1 \cdot 6,-1 \cdot 0$ & \\
\hline Waist circumference (per $1 \mathrm{~cm}$ increment) & -0.4 & $-0.5,-0.4$ & $0 \cdot 1$ & $0.0,0.3$ & \\
\hline \multicolumn{6}{|l|}{ Education } \\
\hline Low & Ref. & & Ref. & & \\
\hline Medium & $8 \cdot 3$ & $6 \cdot 7,10 \cdot 0$ & $4 \cdot 1$ & $2 \cdot 6,5 \cdot 6$ & \\
\hline High & $23 \cdot 4$ & $21 \cdot 3,25 \cdot 5$ & $13 \cdot 2$ & $11 \cdot 1,15 \cdot 2$ & \\
\hline
\end{tabular}

Ref., reference.

${ }^{*}$ Regression coefficients $(\beta)$ reflect the difference in the intake of wholegrain products $(\mathrm{g} / \mathrm{d})$ for each given increment in the independent variable. tAmong current drinkers.

¥Current drinkers with median intake of $20 \mathrm{~g}$ alcohol $/ \mathrm{d}$.

$\S$ Variation in intake of wholegrain products explained by dietary variables.

IIVariation in intake of wholegrain products explained by dietary and physical activity variables.

TVariation in intake of wholegrain products explained by dietary, physical activity, lifestyle (including anthropometry) and socio-economic variables.

school education and negative associations with alcohol intake, BMI and smoking were observed among both men and women.

Although the participants in the present study are high consumers of wholegrain products, these results still mirror findings of previous studies on populations with different consumption patterns. In the Iowa Women's Health Study a higher intake of whole grains was associated with higher education, lower BMI and waist:hip ratio, being a non-smoker, doing more regular physical 
Table 4 Regression derived coefficients $(\beta)$ and $95 \%$ confidence intervals of dietary, lifestyle, anthropometric and socio-economic variables among 26175 men included in the Diet, Cancer and Health cohort study (1993-7)

\begin{tabular}{|c|c|c|c|c|c|}
\hline & \multicolumn{2}{|c|}{ Analysis adjusted for age and centre } & \multicolumn{2}{|c|}{ Mutual adjusted analysis } & \multirow[b]{2}{*}{$R^{2}$} \\
\hline & $\beta^{*}$ & $95 \% \mathrm{Cl}$ & $\beta^{\star}$ & $95 \% \mathrm{Cl}$ & \\
\hline Dietary variables & & & & & $0 \cdot 15 \S$ \\
\hline Fish (per $25 \mathrm{~g}$ ) & $9 \cdot 4$ & $8 \cdot 7,10 \cdot 2$ & $4 \cdot 7$ & $4 \cdot 0,5 \cdot 5$ & \\
\hline Red meat (per $50 \mathrm{~g}$ ) & $-2 \cdot 2$ & $-3 \cdot 2,-1 \cdot 2$ & $-10 \cdot 1$ & $-11 \cdot 1,-9 \cdot 1$ & \\
\hline Poultry (per $10 \mathrm{~g}$ ) & $3 \cdot 4$ & $2 \cdot 9,3 \cdot 8$ & $0 \cdot 7$ & $0 \cdot 3,1 \cdot 1$ & \\
\hline Processed meat (per $10 \mathrm{~g}$ ) & $3 \cdot 7$ & $3 \cdot 4,4 \cdot 1$ & $6 \cdot 0$ & $5 \cdot 7,6 \cdot 3$ & \\
\hline Lean dairy products (per $100 \mathrm{~g}$ ) & $2 \cdot 3$ & $2 \cdot 0,2 \cdot 6$ & $1 \cdot 2$ & $1 \cdot 0,1 \cdot 5$ & \\
\hline Fatty dairy products (per $50 \mathrm{~g}$ ) & $1 \cdot 3$ & $1 \cdot 1,1 \cdot 6$ & $0 \cdot 8$ & $0 \cdot 5,1 \cdot 0$ & \\
\hline Fruits (per $100 \mathrm{~g}$ ) & $7 \cdot 4$ & $6 \cdot 8,8 \cdot 0$ & $1 \cdot 8$ & $1 \cdot 3,2 \cdot 4$ & \\
\hline Vegetables (per $100 \mathrm{~g}$ ) & $18 \cdot 0$ & $17 \cdot 1,18 \cdot 9$ & $12 \cdot 9$ & $11 \cdot 9,13 \cdot 9$ & \\
\hline Cakes (per $10 \mathrm{~g}$ ) & $2 \cdot 0$ & $1 \cdot 6,2 \cdot 3$ & $1 \cdot 9$ & $1 \cdot 6,2 \cdot 3$ & \\
\hline Refined grain products (per $30 \mathrm{~g}$ ) & $-6 \cdot 8$ & $-7 \cdot 4,-6 \cdot 1$ & $-11 \cdot 7$ & $-12 \cdot 4,-11 \cdot 1$ & \\
\hline Physical activity variables & & & & & $0 \cdot 17 \|$ \\
\hline Walking (per $1 \mathrm{~h}$ ) & $2 \cdot 2$ & $-0 \cdot 3,4 \cdot 8$ & $-0 \cdot 3$ & $-2 \cdot 6,2 \cdot 1$ & \\
\hline Cycling (per $1 \mathrm{~h}$ ) & $17 \cdot 1$ & $14 \cdot 5,19 \cdot 8$ & $10 \cdot 9$ & $8 \cdot 4,13 \cdot 4$ & \\
\hline Sports (per $1 \mathrm{~h}$ ) & $6 \cdot 3$ & $2 \cdot 6,10 \cdot 0$ & $2 \cdot 8$ & $-0.5,6 \cdot 1$ & \\
\hline Housework (per $1 \mathrm{~h}$ ) & $3 \cdot 4$ & $0 \cdot 4,6 \cdot 3$ & $2 \cdot 4$ & $-0 \cdot 3,5 \cdot 2$ & \\
\hline Gardening (per $1 \mathrm{~h}$ ) & $2 \cdot 2$ & $-0 \cdot 4,4 \cdot 9$ & $-1 \cdot 8$ & $-4 \cdot 4,0 \cdot 7$ & \\
\hline Hobby work (per $1 \mathrm{~h})$ & $4 \cdot 8$ & $2 \cdot 3,7 \cdot 4$ & $2 \cdot 3$ & $-0 \cdot 2,4 \cdot 8$ & \\
\hline Lifestyle and socio-economic variables & & & & & $0 \cdot 21 \uparrow$ \\
\hline \multicolumn{6}{|l|}{ Use of fatty substance when cooking } \\
\hline None & Ref. & & Ref. & & \\
\hline Vegetable oil & $-18 \cdot 4$ & $-34 \cdot 1,-2 \cdot 7$ & $-8 \cdot 4$ & $-22 \cdot 8,5 \cdot 8$ & \\
\hline Margarine/butter & $-33 \cdot 7$ & $-49 \cdot 3,-18 \cdot 1$ & $-14 \cdot 5$ & $-28 \cdot 7,-0 \cdot 2$ & \\
\hline \multicolumn{6}{|l|}{ Use of dietary supplement } \\
\hline Non-user & Ref. & & Ref. & & \\
\hline Multivitamin/mineral user & $13 \cdot 6$ & $11 \cdot 1,16 \cdot 2$ & $5 \cdot 4$ & $3 \cdot 2,7 \cdot 7$ & \\
\hline Any user & $16 \cdot 9$ & $15 \cdot 0,18 \cdot 9$ & $6 \cdot 9$ & $5 \cdot 1,8 \cdot 7$ & \\
\hline Alcohol intaket (per $10 \mathrm{~g})$ & $-4 \cdot 3$ & $-4 \cdot 7,-4 \cdot 0$ & $-2 \cdot 8$ & $-3 \cdot 1,-2 \cdot 5$ & \\
\hline \multicolumn{6}{|l|}{ Alcohol status } \\
\hline Never drinker & Ref. & & Ref. & & \\
\hline Ex-drinker & $2 \cdot 8$ & $-17 \cdot 6,23 \cdot 2$ & $-1 \cdot 1$ & $-19 \cdot 0,16 \cdot 8$ & \\
\hline Occasional drinker & $-1 \cdot 1$ & $-21 \cdot 8,19 \cdot 6$ & $-1 \cdot 1$ & $-23 \cdot 9,21 \cdot 7$ & \\
\hline Current drinker‡ & $-6 \cdot 0$ & $-24 \cdot 9,12 \cdot 9$ & $-2 \cdot 4$ & $-26 \cdot 8,21 \cdot 9$ & \\
\hline \multicolumn{6}{|l|}{ Alcohol preferencet } \\
\hline Wine drinker & Ref. & & Ref. & & \\
\hline Beer drinker & $-11 \cdot 1$ & $-13 \cdot 2,-9 \cdot 1$ & $-2 \cdot 8$ & $-4 \cdot 8,-0.9$ & \\
\hline Spirits drinker & $-6 \cdot 8$ & $-12 \cdot 6,-1 \cdot 0$ & $-2 \cdot 2$ & $-7 \cdot 4,2 \cdot 9$ & \\
\hline Mixed drinker & $0 \cdot 1$ & $-2 \cdot 4,2 \cdot 7$ & -0.3 & $-2 \cdot 5,2 \cdot 0$ & \\
\hline \multicolumn{6}{|l|}{ Smoking status } \\
\hline Never smoker & Ref. & & Ref. & & \\
\hline Past smoker & $-2 \cdot 7$ & $-5 \cdot 0,-0 \cdot 4$ & $1 \cdot 0$ & $-1 \cdot 0,3 \cdot 1$ & \\
\hline Current smoker & $-15 \cdot 2$ & $-17 \cdot 4,-12 \cdot 9$ & $-6 \cdot 4$ & $-8 \cdot 4,-4 \cdot 3$ & \\
\hline BMI (per $1 \mathrm{~kg} / \mathrm{m}^{2}$ increment) & $-2 \cdot 9$ & $-3 \cdot 2,-2 \cdot 7$ & $-2 \cdot 2$ & $-2 \cdot 7,-1 \cdot 7$ & \\
\hline Waist circumference (per $1 \mathrm{~cm}$ increment) & $-1 \cdot 1$ & $-1 \cdot 2,-1 \cdot 0$ & -0.2 & $-0 \cdot 4,-0 \cdot 1$ & \\
\hline \multicolumn{6}{|l|}{ Education } \\
\hline Low & Ref. & & Ref. & & \\
\hline Medium & $1 \cdot 2$ & $-0.8,3 \cdot 3$ & $-2 \cdot 1$ & $-4 \cdot 0,-0 \cdot 2$ & \\
\hline High & $12 \cdot 9$ & $10 \cdot 6,15 \cdot 3$ & $3 \cdot 4$ & $1 \cdot 1,5 \cdot 7$ & \\
\hline
\end{tabular}

Ref., reference.

${ }^{*}$ Regression coefficients $(\beta)$ reflect the difference in the intake of wholegrain products $(\mathrm{g} / \mathrm{d})$ for each given increment in the independent variable. tAmong current drinkers.

¥Current drinkers with median intake of $10 \mathrm{~g}$ alcohol $/ \mathrm{d}$.

\&Variation in intake of wholegrain products explained by dietary variables.

IIVariation in intake of wholegrain products explained by dietary and physical activity variables.

TVariation in intake of wholegrain products explained by dietary, physical activity, lifestyle (including anthropometry) and socio-economic variables.

activity, using vitamin supplements and HRT. Higher whole grains intake was also associated with greater energy intake, consumption of less refined grains, sucrose and red meat, and consumption of more fruits and vegetables ${ }^{(29)}$. Additionally, surveys performed in the USA and UK have shown that consumers of whole grains are more often non-smokers, regular exercisers and consume more fruits and vegetables ${ }^{(30,31)}$. In a Norwegian study it was concluded that Norwegian wholegrain bread eaters were less likely to be smokers and were more physically active than white bread eaters, and in addition had lower serum cholesterol and systolic blood pressure and ate less total and saturated fat as a proportion of energy intake $^{(3)}$. 
Table 5 Mutual adjusted regression analysis derived coefficients $(\beta)$, standard errors, $R^{2}$ and $P$ values of dietary patterns ${ }^{*}, \dagger$ (ten principal components (PC)) for the intake of wholegrain products among 26175 men and 28545 women included in the Diet, Cancer and Health cohort study (1993-7)

\begin{tabular}{|c|c|c|c|c|c|c|c|c|c|c|}
\hline & \multicolumn{10}{|c|}{ Women } \\
\hline & $\mathrm{PC} 1$ & PC10 & PC2 & PC8 & PC9 & PC5 & PC3 & PC6 & PC7 & PC4 \\
\hline \multirow{7}{*}{$\begin{array}{l}\text { Intake of wholegrain products }(\mathrm{g} / \mathrm{d}) \\
R^{2} \\
\beta \ddagger \\
\mathrm{SE} \\
P \text { value }\end{array}$} & & & & & & & & & & \\
\hline & 0.0323 & 0.0616 & $0 \cdot 0845$ & 0.0999 & $0 \cdot 1089$ & $0 \cdot 1171$ & 0.1197 & $0 \cdot 1203$ & 0.1209 & $0 \cdot 1209$ \\
\hline & $7 \cdot 1$ & $13 \cdot 6$ & $-5 \cdot 1$ & $9 \cdot 3$ & $5 \cdot 9$ & $5 \cdot 1$ & $0 \cdot 2$ & $1 \cdot 5$ & & $-1 \cdot 2$ \\
\hline & $0 \cdot 2$ & 0.5 & $0 \cdot 3$ & $0 \cdot 4$ & 0.4 & & $0 \cdot 2$ & 0.4 & & 0.3 \\
\hline & $<10^{-3}$ & $<10^{-3}$ & $<10^{-3}$ & $<10^{-3}$ & $<10^{-3}$ & $<10^{-3}$ & $0 \cdot 491$ & $<10^{-3}$ & $<10^{-3}$ & $<10^{-3}$ \\
\hline & \multicolumn{10}{|c|}{ Men } \\
\hline & PC10 & PC1 & PC5 & PC2 & PC8 & PC9 & PC6 & PC3 & PC7 & PC4 \\
\hline \multicolumn{11}{|l|}{ Intake of wholegrain products $(\mathrm{g} / \mathrm{d})$} \\
\hline & 0.0613 & 0.0964 & $0 \cdot 1183$ & $0 \cdot 1318$ & $0 \cdot 1449$ & $0 \cdot 1514$ & $0 \cdot 1516$ & $0 \cdot 1517$ & $0 \cdot 1517$ & $0 \cdot 1517$ \\
\hline$\beta \ddagger$ & $21 \cdot 6$ & $8 \cdot 4$ & $10 \cdot 8$ & $-4 \cdot 9$ & $10 \cdot 6$ & $6 \cdot 7$ & $-1 \cdot 6$ & $-3 \cdot 3$ & $0 \cdot 1$ & -0.9 \\
\hline SE & $0 \cdot 6$ & $0 \cdot 3$ & $0 \cdot 4$ & $0 \cdot 4$ & 0.5 & & 0.5 & $0 \cdot 4$ & 0.5 & 0.4 \\
\hline$P$ value & $<10^{-3}$ & $<10^{-3}$ & $<10^{-3}$ & $<10^{-3}$ & $<10^{-3}$ & $<10^{-3}$ & $<10^{-3}$ & $<10^{-3}$ & 0.859 & 0.0025 \\
\hline
\end{tabular}

*See Appendix 1 and 2 for description.

tPrincipal components are presented in descending order of how much of the total variation in the intake of wholegrain products they explain $\left(R^{2}\right)$. †Mutual adjusted and adjusted for age, centre, alcohol intake, alcohol preference, alcohol status, physical activity (hours per week spent walking, hours per week spent cycling, hours per week spent doing sports, hours per week spent gardening, hours per week spent doing housework, hours per week spent doing hobby work), intake of dietary supplements, smoking status, school education, use of a fatty substance when cooking, BMI, waist circumference and hormone replacement therapy (women only).

Dietary factors may cluster together; thus instead of looking at individual foods or nutrients, dietary pattern analysis has recently emerged as an alternative and complementary approach in the study of diet in relation to diseases ${ }^{(38)}$. In addition to the single nutrient approach, we used principal components analysis to extract dietary patterns from the ten food groups studied and evaluated these in association to intake of wholegrain products. We observed that different dietary patterns were differently related to intake of wholegrain products. In both men and women, the strongest positive associations with intake of wholegrain products were seen for a dietary pattern (PC1) that could be interpreted as a 'varied' dietary pattern because of positive loadings for all dietary factors studied and a dietary pattern (PC10) that was characterized by a contrast between processed meat and vegetables (positive scoring coefficients) and red meat and refined-grain products (negative scoring coefficients). These two dietary patterns explained more than half of the variation in the total intake of wholegrain products among men $(9 \cdot 6 \%)$ and women $(6 \cdot 2 \%)$. In the present study, describing the diet in combination and relating this to the intake of wholegrain products yielded results that were comparable to the results obtained in regression analyses focusing on individual dietary factors, indicating that the study of dietary patterns could be a useful alternative when evaluating the association between wholegrain products and other dietary factors.

There is growing epidemiological evidence indicating that whole grains or wholegrain foods reduce the risk of several chronic diseases including $\mathrm{CVD}^{(2,5-9)}$, type 2 diabetes $^{(10-14)}$ and some cancers ${ }^{(15-17)}$. Whole grains contain many bioactive compounds that might be responsible for their protective effects; e.g. fermentable carbohydrates that affect the gut environment, compounds that function as antioxidants (e.g. trace minerals and phenolic compounds) and phyto-oestrogens with potential hormonal effects ${ }^{(28)}$. Alternatively, the protective effects of whole grains and wholegrain foods towards the development of chronic diseases could be mediated via their beneficial effect on weight control through the promotion of satiety and enhancement of satiation ${ }^{(28)}$. In the present study, higher wholegrain products intake was associated with lower BMI, which is comparable to findings from other crosssectional studies ${ }^{(18-21)}$. Also, risk of obesity or abdominal obesity has cross-sectionally been reported to be lower among persons with high intakes of whole grains compared with persons with low intakes ${ }^{(25)}$. In addition, in prospective studies intake (or change in intake) of whole grains has been inversely associated with weight gain or gain in $\mathrm{BMI}^{(22-24)}$. Cross-sectionally, high intakes of whole grains have been linked to less frequent development of metabolic syndrome ${ }^{(25-27)}$, a risk factor of CVD and type 2 diabetes. Epidemiological studies have also indicated that persons with the metabolic syndrome are at increased risk of colon cancer ${ }^{(39)}$.

The strengths of the present study are its large size and that data on dietary intake were measured by a validated $\mathrm{FFQ}^{(34-36)}$. Measurements of alcohol habits, smoking status and physical activity in leisure time were also collected by a comprehensive lifestyle questionnaire. At baseline, anthropometric measurements (height, weight, waist circumference) were measured by trained health professionals. We used data from a large population with a high degree of variability in the intake of wholegrain products, which allowed us to consider a number of 
different factors altogether. There are several limitations to our study. First, our study is cross-sectional. Second, we cannot exclude that measurement errors introduced by either the participants or the questionnaires may have affected the results. In spite of the comprehensive model tested in the present study, including several dietary, lifestyle and socio-economic factors hypothesized to be associated with intake of wholegrain products, only $16 \%$ and $21 \%$ of the variation in the intake of wholegrain products among women and men, respectively, was explained by the model. The inability to explain a higher percentage of the variance in wholegrain products intake may be due to several factors, including difficulties inherent in the assessing of wholegrain products and an inability to measure unknown factors relevant to the intake of wholegrain products.

In conclusion, the present results suggest that consumption of wholegrain products is associated with other individual dietary factors and dietary patterns, healthier lifestyle habits and higher socio-economic status. This could contribute to the associations observed between wholegrain products intake and risk of various chronic diseases. Future epidemiological studies on the intake of wholegrain products and disease risk need to take into account possible confounding by other dietary and lifestyle-related parameters when examining the relationship between wholegrain products intake and risk of diseases.

\section{Acknowledgements}

Sources of funding: The study was funded by the Danish Council for Strategic Research, Programme Commission on Food and Health. Conflict of interest declaration: There are no conflicts of interest to report. Authorship responsibilities: A.T. and K.O. contributed 'to the original design and data collection for the Diet, Cancer and Health project. R.E. was responsible for planning the analysis strategy, but all authors contributed to the process. R.E. performed the statistical analyses under the supervision of K.F. and drafted the manuscript. All authors (R.E., A.T., K.O., K.F., A.O., N.F.J., S.L.) contributed to the interpretation of the results and made critical comments during the preparation of the manuscript. Acknowledgements: The work was performed at the Institute of Cancer Epidemiology, The Danish Cancer Society. We thank Katja Boll (data manager) and Jytte Fogh Larsen (project coordinator) for assistance with the data collection and Connie Stripp (dietitian) for assistance with the dietary data.

\section{References}

1. Liu S, Sesso HD, Manson JE, Willett WC \& Buring JE (2003) Is intake of breakfast cereals related to total and causespecific mortality in men? Am J Clin Nutr 77, 594-599.
2. Steffen LM, Jacobs DR Jr, Stevens J, Shahar E, Carithers T \& Folsom AR (2003) Associations of whole-grain, refinedgrain, and fruit and vegetable consumption with risks of all-cause mortality and incident coronary artery disease and ischemic stroke: the Atherosclerosis Risk in Communities (ARIC) Study. Am J Clin Nutr 78, 383-390.

3. Jacobs DR Jr, Meyer HE \& Solvoll K (2001) Reduced mortality among whole grain bread eaters in men and women in the Norwegian County Study. EurJ Clin Nutr $\mathbf{5 5}$ $137-143$.

4. Jacobs DR Jr, Meyer KA, Kushi LH \& Folsom AR (1999) Is whole grain intake associated with reduced total and cause-specific death rates in older women? The Iowa Women's Health Study. Am J Public Health 89, 322-329.

5. Jensen MK, Koh-Banerjee P, Hu FB, Franz M, Sampson L, Gronbaek M \& Rimm EB (2004) Intakes of whole grains, bran, and germ and the risk of coronary heart disease in men. Am J Clin Nutr 80, 1492-1499.

6. Liu S, Stampfer MJ, Hu FB, Giovannucci E, Rimm E, Manson JE, Hennekens CH \& Willett WC (1999) Whole-grain consumption and risk of coronary heart disease: results from the Nurses' Health Study. Am J Clin Nutr 70, 412-419.

7. Fraser GE (1999) Associations between diet and cancer, ischemic heart disease, and all-cause mortality in nonHispanic white California Seventh-day Adventists. Am J Clin Nutr 70, 532S-538S.

8. Liu S, Manson JE, Stampfer MJ, Rexrode KM, Hu FB, Rimm EB \& Willett WC (2000) Whole grain consumption and risk of ischemic stroke in women: a prospective study. JAMA 284, 1534-1540.

9. Mellen PB, Walsh TF \& Herrington DM (2007) Whole grain intake and cardiovascular disease: a meta-analysis. Nutr Metab Cardiovasc Dis 18, 283-290.

10. Montonen J, Knekt P, Jarvinen R, Aromaa A \& Reunanen A (2003) Whole-grain and fiber intake and the incidence of type 2 diabetes. Am J Clin Nutr 77, 622-629.

11. Fung TT, Hu FB, Pereira MA, Liu S, Stampfer MJ, Colditz GA \& Willett WC (2002) Whole-grain intake and the risk of type 2 diabetes: a prospective study in men. Am J Clin Nutr $\mathbf{7 6}$, $535-540$.

12. Liu S, Manson JE, Stampfer MJ, Hu FB, Giovannucci E, Colditz GA, Hennekens CH \& Willett WC (2000) A prospective study of whole-grain intake and risk of type 2 diabetes mellitus in US women. Am J Public Health 90, 1409-1415.

13. Meyer KA, Kushi LH, Jacobs DR Jr, Slavin J, Sellers TA \& Folsom AR (2000) Carbohydrates, dietary fiber, and incident type 2 diabetes in older women. Am J Clin Nutr 71, 921-930.

14. de Munter JS, Hu FB, Spiegelman D, Franz M \& van Dam RM (2007) Whole grain, bran, and germ intake and risk of type 2 diabetes: a prospective cohort study and systematic review. PLoS Med 4, e261.

15. Larsson SC, Giovannucci E, Bergkvist L \& Wolk A (2005) Whole grain consumption and risk of colorectal cancer: a population-based cohort of 60,000 women. Br J Cancer $\mathbf{9 2 ,}$ 1803-1807.

16. Kasum CM, Jacobs DR Jr, Nicodemus K \& Folsom AR (2002) Dietary risk factors for upper aerodigestive tract cancers. Int J Cancer 99, 267-272.

17. Schatzkin A, Mouw T, Park Y, Subar AF, Kipnis V, Hollenbeck A, Leitzmann MF \& Thompson FE (2007) Dietary fiber and whole-grain consumption in relation to colorectal cancer in the NIH-AARP Diet and Health Study. Am J Clin Nutr 85, 1353-1360.

18. Thane CW, Jones AR, Stephen AM, Seal CJ \& Jebb SA (2005) Whole-grain intake of British young people aged 4-18 years. Br J Nutr 94, 825-831.

19. Steffen LM, Jacobs DR Jr, Murtaugh MA, Moran A, Steinberger J, Hong CP \& Sinaiko AR (2003) Whole grain intake is 
associated with lower body mass and greater insulin sensitivity among adolescents. Am J Epidemiol 158, 243-250.

20. van de Vijver LP, van den Bosch LM, van den Brandt PA, Goldbohm RA (2007) Whole-grain consumption, dietary fibre intake and body mass index in the Netherlands cohort study. Eur J Clin Nutr (Epublication ahead of print version).

21. McKeown NM, Meigs JB, Liu S, Wilson PW \& Jacques PF (2002) Whole-grain intake is favorably associated with metabolic risk factors for type 2 diabetes and cardiovascular disease in the Framingham Offspring Study. Am J Clin Nutr 76, 390-398.

22. Bazzano LA, Song Y, Bubes V, Good CK, Manson JE \& Liu S (2005) Dietary intake of whole and refined grain breakfast cereals and weight gain in men. Obes Res 13, 1952-1960.

23. Koh-Banerjee P, Franz M, Sampson L, Liu S, Jacobs DR Jr, Spiegelman D, Willett W \& Rimm E (2004) Changes in whole-grain, bran, and cereal fiber consumption in relation to 8-y weight gain among men. Am J Clin Nutr 80, $1237-1245$.

24. Liu S, Willett WC, Manson JE, Hu FB, Rosner B \& Colditz G (2003) Relation between changes in intakes of dietary fiber and grain products and changes in weight and development of obesity among middle-aged women. Am J Clin Nutr 78, 920-927.

25. Esmaillzadeh A, Mirmiran P \& Azizi F (2005) Whole-grain consumption and the metabolic syndrome: a favorable association in Tehranian adults. Eur J Clin Nutr 59, 353-362.

26. Sahyoun NR, Jacques PF, Zhang XL, Juan W \& McKeown NM (2006) Whole-grain intake is inversely associated with the metabolic syndrome and mortality in older adults. Am J Clin Nutr 83, 124-131.

27. McKeown NM, Meigs JB, Liu S, Saltzman E, Wilson PW \& Jacques PF (2004) Carbohydrate nutrition, insulin resistance, and the prevalence of the metabolic syndrome in the Framingham Offspring Cohort. Diabetes Care 27, 538-546.

28. Slavin J (2003) Why whole grains are protective: biological mechanisms. Proc Nutr Soc 62, 129-134.

29. Jacobs DR Jr, Meyer KA, Kushi LH \& Folsom AR (1998) Whole-grain intake may reduce the risk of ischemic heart disease death in postmenopausal women: the Iowa Women's Health Study. Am J Clin Nutr 68, 248-257.

30. Lang R, Thane CW, Bolton-Smith C \& Jebb SA (2003) Consumption of whole-grain foods by British adults: findings from further analysis of two national dietary surveys. Public Health Nutr 6, 479-484.

31. Cleveland LE, Moshfegh AJ, Albertson AM \& Goldman JD (2000) Dietary intake of whole grains. J Am Coll Nutr 19, Suppl., 331S-338S.

32. Tjonneland A, Olsen A, Boll K, Stripp C, Christensen J, Engholm G \& Overvad K (2007) Study design, exposure variables, and socioeconomic determinants of participation in Diet, Cancer and Health: a population-based prospective cohort study of 57,053 men and women in Denmark. Scand J Public Health 35, 432-441.

33. Overvad K, Tjonneland A, Haraldsdottir J, Ewertz M \& Jensen OM (1991) Development of a semiquantitative food frequency questionnaire to assess food, energy and nutrient intake in Denmark. Int J Epidemiol 20, 900-905.

34. Tjonneland A, Overvad K, Haraldsdottir J, Bang S, Ewertz M \& Jensen OM (1991) Validation of a semiquantitative food frequency questionnaire developed in Denmark. Int J Epidemiol 20, 906-912.

35. Tjonneland A, Haraldsdottir J, Overvad K, Stripp C, Ewertz M \& Jensen OM (1992) Influence of individually estimated portion size data on the validity of a semiquantitative food frequency questionnaire. Int J Epidemiol 21, 770-777.

36. Haraldsdottir J, Tjonneland A \& Overvad K (1994) Validity of individual portion size estimates in a food frequency questionnaire. Int J Epidemiol 23, 786-796.

37. Greenland S (1995) Dose-response and trend analysis in epidemiology: alternatives to categorical analysis. Epidemiology 6, 356-365.

38. Hu FB (2002) Dietary pattern analysis: a new direction in nutritional epidemiology. Curr Opin Lipidol 13, 3-9.

39. Giovannucci E (2007) Metabolic syndrome, hyperinsulinemia, and colon cancer: a review. Am J Clin Nutr 86, Suppl., s836-s842. 
Appendix 1

Principal components and corresponding scoring coefficients for dietary variables among 28545 women in the Diet, Cancer and Health cobort study

\begin{tabular}{|c|c|c|c|}
\hline Principal component & Positive scoring coefficients & Negative scoring coefficients & Variance explained (\%) \\
\hline $\mathrm{PC} 1$ & $\begin{array}{l}\text { Fish }(0 \cdot 45) \\
\text { Red meat }(0 \cdot 43) \\
\text { Poultry }(0 \cdot 32) \\
\text { Processed meat }(0 \cdot 32) \\
\text { Lean dairy products }(0 \cdot 17) \\
\text { Fatty dairy products }(0 \cdot 16) \\
\text { Fruits }(0 \cdot 32) \\
\text { Vegetables }(0 \cdot 35) \\
\text { Cakes }(0 \cdot 20) \\
\text { Refined-grain products }(0 \cdot 29)\end{array}$ & & 20 \\
\hline PC2 & $\begin{array}{l}\text { Red meat }(0 \cdot 27) \\
\text { Processed meat }(0 \cdot 43) \\
\text { Fatty dairy products }(0 \cdot 12) \\
\text { Cakes }(0 \cdot 38) \\
\text { Refined-grain products }(0 \cdot 38)\end{array}$ & $\begin{array}{l}\text { Fish }(-0.20) \\
\text { Poultry }(-0.28) \\
\text { Lean dairy products }(-0.09) \\
\text { Fruits }(-0.33) \\
\text { Vegetables }(-0.46)\end{array}$ & 16 \\
\hline PC3 & $\begin{array}{l}\text { Fatty dairy products }(0 \cdot 39) \\
\text { Fruits }(0 \cdot 29) \\
\text { Vegetables }(0 \cdot 17) \\
\text { Cakes }(0 \cdot 50) \\
\text { Refined-grain products }(0 \cdot 34)\end{array}$ & $\begin{array}{l}\text { Fish }(-0.09) \\
\text { Red meat }(-0.37) \\
\text { Poultry }(-0.15) \\
\text { Processed meat }(-0.40) \\
\text { Lean dairy products }(-0.21)\end{array}$ & 11 \\
\hline PC4 & $\begin{array}{l}\text { Poultry }(0.02) \\
\text { Lean dairy products }(0.63) \\
\text { Fruits }(0.06) \\
\text { Cakes }(0.32) \\
\text { Refined-grain products }(0.24)\end{array}$ & $\begin{array}{l}\text { Fish }(-0.08) \\
\text { Red meat }(-0.18) \\
\text { Processed meat }(-0.11) \\
\text { Fatty dairy products }(-0.62) \\
\text { Vegetables }(-0.06)\end{array}$ & 10 \\
\hline PC5 & $\begin{array}{l}\text { Processed meat }(0 \cdot 14) \\
\text { Vegetables }(0 \cdot 02) \\
\text { Lean dairy products }(0 \cdot 59) \\
\text { Fatty dairy products }(0 \cdot 44) \\
\text { Fruit }(0 \cdot 26)\end{array}$ & $\begin{array}{l}\text { Fish }(-0.00) \\
\text { Red meat }(-0.02) \\
\text { Poultry }(-0.54) \\
\text { Vegetables }(-0.06) \\
\text { Cakes }(-0.13) \\
\text { Refined-grain products }(-0.25)\end{array}$ & 10 \\
\hline PC6 & $\begin{array}{l}\text { Fish }(0 \cdot 26) \\
\text { Poultry }(0 \cdot 34) \\
\text { Lean dairy products }(0 \cdot 36) \\
\text { Fatty dairy products }(0 \cdot 42) \\
\text { Refined-grain products }(0 \cdot 13)\end{array}$ & $\begin{array}{l}\text { Red meat }(-0.22) \\
\text { Processed meat }(-0.20) \\
\text { Fruits }(-0.63) \\
\text { Vegetables }(-0.08) \\
\text { Cakes }(-0.04)\end{array}$ & 8 \\
\hline PC7 & $\begin{array}{l}\text { Poultry }(0 \cdot 55) \\
\text { Processed meat }(0 \cdot 17) \\
\text { Lean dairy products }(0 \cdot 16) \\
\text { Fatty dairy products }(0 \cdot 24) \\
\text { Fruits }(0 \cdot 21)\end{array}$ & $\begin{array}{l}\text { Fish }(-0.73) \\
\text { Red meat }(-0.02) \\
\text { Vegetables }(-0.05) \\
\text { Cakes }(-0.06) \\
\text { Refined-grain products }(-0.01)\end{array}$ & 7 \\
\hline PC8 & $\begin{array}{l}\text { Fish }(0.20) \\
\text { Poultry }(0.24) \\
\text { Processed meat }(0.05) \\
\text { Fatty dairy products }(0.03) \\
\text { Fruits }(0.07) \\
\text { Cakes }(0.59)\end{array}$ & $\begin{array}{l}\text { Red meat }(-0.04) \\
\text { Lean dairy products }(-0.04) \\
\text { Vegetables }(-0.31) \\
\text { Refined-grain products }(-0.67)\end{array}$ & 7 \\
\hline PC9 & $\begin{array}{l}\text { Red meat }(0.36) \\
\text { Lean dairy products }(0.09) \\
\text { Fatty dairy products }(0.00) \\
\text { Vegetables }(0.62) \\
\text { Cakes }(0.30)\end{array}$ & $\begin{array}{l}\text { Fish }(-0.33) \\
\text { Poultry }(-0.17) \\
\text { Processed meat }(-0.14) \\
\text { Fruits }(-0.40) \\
\text { Refined-grain products }(-0.27)\end{array}$ & 6 \\
\hline PC10 & $\begin{array}{l}\text { Fish }(0.03) \\
\text { Processed meat }(0.65) \\
\text { Vegetables }(0.38) \\
\text { Cakes }(0.06)\end{array}$ & $\begin{array}{l}\text { Red meat }(-0.62) \\
\text { Poultry }(-0.05) \\
\text { Lean dairy products }(-0.08) \\
\text { Fatty dairy products }(-0.06) \\
\text { Fruits }(-0.15) \\
\text { Refined-grain products }(-0.06)\end{array}$ & 5 \\
\hline
\end{tabular}

PC, principal component. 
Appendix 2

Principal components and corresponding scoring coefficients for dietary variables among 26175 men in the Diet, Cancer and Health cobort study

\begin{tabular}{|c|c|c|c|}
\hline Principal component & Positive scoring coefficients & Negative scoring coefficients & Variance explained (\%) \\
\hline PC1 & $\begin{array}{l}\text { Fish }(0 \cdot 43) \\
\text { Red meat }(0 \cdot 43) \\
\text { Poultry }(0 \cdot 36) \\
\text { Processed meat }(0 \cdot 25) \\
\text { Lean dairy products }(0 \cdot 12) \\
\text { Fatty dairy products }(0 \cdot 12) \\
\text { Fruits }(0 \cdot 31) \\
\text { Vegetables }(0 \cdot 43) \\
\text { Cakes }(0 \cdot 22) \\
\text { Refined-grain products }(0.29)\end{array}$ & & 19 \\
\hline PC2 & $\begin{array}{l}\text { Red meat }(0.22) \\
\text { Processed meat }(0.47) \\
\text { Fatty dairy products }(0.22) \\
\text { Cakes }(0 \cdot 40) \\
\text { Refined-grain products }(0.40)\end{array}$ & $\begin{array}{l}\text { Fish }(-0 \cdot 17) \\
\text { Poultry }(-0 \cdot 30) \\
\text { Lean dairy products }(-0 \cdot 10) \\
\text { Fruits }(-0.28) \\
\text { Vegetables }(-0.38)\end{array}$ & 15 \\
\hline PC3 & $\begin{array}{l}\text { Fatty dairy products }(0.32) \\
\text { Fruits }(0 \cdot 41) \\
\text { Vegetables }(0 \cdot 12) \\
\text { Cakes }(0 \cdot 47) \\
\text { Refined-grain products }(0 \cdot 27)\end{array}$ & $\begin{array}{l}\text { Fish }(-0 \cdot 15) \\
\text { Red meat }(-0 \cdot 40) \\
\text { Poultry }(-0 \cdot 10) \\
\text { Processed meat }(-0 \cdot 42) \\
\text { Lean dairy products }(-0.22)\end{array}$ & 11 \\
\hline PC4 & $\begin{array}{l}\text { Lean dairy products }(0 \cdot 69) \\
\text { Fruits }(0 \cdot 14) \\
\text { Cakes }(0 \cdot 27) \\
\text { Refined-grain products }(0 \cdot 22)\end{array}$ & $\begin{array}{l}\text { Fish }(-0.14) \\
\text { Red meat }(-0.16) \\
\text { Poultry }(-0.04) \\
\text { Processed meat }(-0.01) \\
\text { Fatty dairy products }(-0.57) \\
\text { Vegetables }(-0.09)\end{array}$ & 11 \\
\hline PC5 & $\begin{array}{l}\text { Fish }(0.04) \\
\text { Red meat }(0.02) \\
\text { Processed meat }(0.18) \\
\text { Lean dairy products }(0.49) \\
\text { Fatty dairy products }(0.51) \\
\text { Fruits }(0.37)\end{array}$ & $\begin{array}{l}\text { Poultry }(-0.43) \\
\text { Vegetables }(-0.04) \\
\text { Cakes }(-0.13) \\
\text { Refined-grain products }(-0.34)\end{array}$ & 9 \\
\hline PC6 & $\begin{array}{l}\text { Fish }(0 \cdot 19) \\
\text { Poultry }(0 \cdot 41) \\
\text { Lean dairy products }(0 \cdot 42) \\
\text { Fatty dairy products }(0 \cdot 47) \\
\text { Cakes }(0 \cdot 03) \\
\text { Refined-grain products }(0 \cdot 13)\end{array}$ & $\begin{array}{l}\text { Red meat }(-0.27) \\
\text { Processed meat }(-0.21) \\
\text { Fruits }(-0.51) \\
\text { Vegetables }(-0.12)\end{array}$ & 8 \\
\hline PC7 & $\begin{array}{l}\text { Red meat }(0 \cdot 01) \\
\text { Poultry }(0 \cdot 43) \\
\text { Processed meat }(0 \cdot 24) \\
\text { Lean dairy products }(0.07) \\
\text { Fatty dairy products }(0 \cdot 18) \\
\text { Fruits }(0 \cdot 15) \\
\text { Vegetables }(0 \cdot 15) \\
\text { Refined-grain products }(0 \cdot 11)\end{array}$ & $\begin{array}{l}\text { Fish }(-0.78) \\
\text { Cakes }(-0.24)\end{array}$ & 7 \\
\hline PC8 & $\begin{array}{l}\text { Fish }(0 \cdot 20) \\
\text { Poultry }(0 \cdot 43) \\
\text { Processed meat }(0 \cdot 40) \\
\text { Fruits }(0 \cdot 32) \\
\text { Cakes }(0 \cdot 18)\end{array}$ & $\begin{array}{l}\text { Red meat }(-0.37) \\
\text { Lean dairy products }(-0.16) \\
\text { Fatty dairy products }(-0.05) \\
\text { Vegetables }(-0.49) \\
\text { Refined-grain products }(-0.29)\end{array}$ & 7 \\
\hline PC9 & $\begin{array}{l}\text { Red meat }(0.33) \\
\text { Poultry }(0 \cdot 14) \\
\text { Lean dairy products }(0.06) \\
\text { Fatty dairy products }(0.01) \\
\text { Vegetables }(0 \cdot 11) \\
\text { Cakes }(0.60)\end{array}$ & $\begin{array}{l}\text { Fish }(-0 \cdot 25) \\
\text { Processed meat }(-0 \cdot 15) \\
\text { Fruits }(-0 \cdot 18) \\
\text { Refined-grain products }(-0.62)\end{array}$ & 7 \\
\hline PC10 & $\begin{array}{l}\text { Fish }(0.03) \\
\text { Processed meat }(0.47) \\
\text { Vegetables }(0.60) \\
\text { Cakes }(0.14)\end{array}$ & $\begin{array}{l}\text { Red meat }(-0.52) \\
\text { Poultry }(-0.17) \\
\text { Lean dairy products }(-0.04) \\
\text { Fatty dairy products }(-0.05) \\
\text { Fruits }(-0.29) \\
\text { Refined-grain products }(-0.13)\end{array}$ & 6 \\
\hline
\end{tabular}

PC, principal component. 